room, stores and service rooms, as well as private rooms for individual research officers. Much thought has gone into the planning of the layout, and the architect has designed a light and airy building of pleasing character, yet retaining its strictly functional purpose, for a total cost of $£ 15,000$.

The Station is situated at 7,100 ft. in the Kenya Highlands, virtually on the equator, in one of the main wheat-growing areas of the country. It is the headquarters of the Plant Breeding Section, and the main programme on wheat, barley and oats is concentrated there. The present staff of the section, including two plant breeders working on maize at subsidiary stations, comprises eight graduate officers and additional supporting staff.

History records that the early settlers in Kenya started growing wheat at the beginning of the century ; but that they suffered great loss due to rust attacks. Although a certain amount of desultory work was carried out from 1910 onwards, it was not until 1928 that wheat-breeding was put on an organized basis as a result of the visit of the late Sir Rowland Biffen, and the advice he gave to the Government to set up a wheat-breeding station. In that year the original station was built at Njoro and the late Capt. J. G. L. Burton, one of Biffen's pupils, was appointed to the first post of plant breeder. He was assisted by Mr. R. J. Lathbury, who later took charge when Burton retired. The Station has, until recently, remained virtually unchanged since those early days.

The present period of expansion dates from 1952-53 when development funds were first made available. The provision of improved facilities had become especially necessary due to the severe outbreaks of rust causing great damage to existing wheats and the urgent need to produce resistant varieties. The development has entailed recruitment of extra staff, purchase of additional land and specialized equipment, and now the construction of the new laboratories. It is especially satisfactory that farmers themselves, by agreeing to a cess on wheat, have made a substantial contribution to this development. Certain further projects, however, connected with rust pathology and maize breeding await the grant of special funds.

The plant breeding team is fortunate in being provided with such excellent new laboratories and modern facilities. Indeed, these are essential to the necessary intensification of the research programme to provide the rust-resistant wheats Kenya so urgently needs.

\title{
THE GALACTIC SYSTEM AS A SPIRAL NEBULA
}

$\mathrm{T}$ HE view that the Galaxy might have a spiral structure has been expressed almost since the first discoveries of such structure in nebulæ. It was not until 1951, however, that part of the galactic spiral structure was actually found. Work in this field has recently been summarized in a report on the progress of astronomy by J. H. Oort, F. T. Kerr and G. Westerhout (Mon. Not. Roy. Astro. Soc., 118, 379 ; 1958). The first definite discovery of spiral structure was accomplished by W. W. Morgan, S. Sharpless and D. E. Osterbrock, using the luminosity criteria for $O$ and $B$ stars developed by Morgan. They determined the distances of groups of these stars, and they showed that the regions of ionized hydrogen excited by the stars were arranged in long chains which formed parts of spiral arms. By a remarkable coincidence, radiation on $21-\mathrm{cm}$. wave-length from interstellar hydrogen was detected at about the same time as the visual work was done, and by 1954 a comprehensive picture of the spiral structure of the Galaxy could be assembled from radio observations. The importance of hydrogen-line radiation at $21 \mathrm{~cm}$. arises partly because of the transparency of inter. stellar dust to radio waves and partly because the study of this radio spectrum line gives discrimination in distance. Actual distances can only be inferred from radial velocities, and this involves the assumptions of circular motion and of equality of motions of stars and gas in any particular region of the Galaxy.

In order to transform radial velocities into distances, we must know how the circular velocity in the Galaxy varies with the distance from the galactic centre, and we must know the distance of the Sun from the centre. If we know the distance and circular velocity of the Sun we can derive, from the radio observations themselves, the variation of circular velocity with distance from the centre for distances smaller than that of the Sun. The distance from the Sun to the centre has been obtained directly from the study of the distribution of cluster-type cepheid variables concentrated near the centre. The best estimate obtained in this way is 8.2 kiloparsecs, the accuracy being limited by the uncertainty in the interstellar absorption and in the absolute magnitude of the variables. The circular velocity, and an independent estimate of the solar distance, can be obtained by combining radio observations with the constants of differential galactic rotation obtained from the proper motions and radial velocities of distant stars. The best available estimate of the circular velocity of the Sun is $215 \mathrm{~km}$. $/ \mathrm{sec}$. For regions of the Galaxy farther from the centre than the Sun we cannot determine the rotational velocities from radio observations, and we must rely on values computed from models of the Galaxy based on stellar densities. The rotation curve derived by $\mathbf{M}$. Schmidt from all available data shows a maximum of about $225 \mathrm{~km}$. $/ \mathrm{sec}$. at a distance of 7 kiloparsecs from the centre.

From profiles of the $21-\mathrm{cm}$. line one can now derive the density distribution of the neutral hydrogen in the Galaxy. Most of the hydrogen is confined to a flat disk, with a half-density whole-width of 220 parsecs. This hydrogen disk is remarkably flat; nowhere within 8 kiloparsecs from the centre do the points of maximum hydrogen density deviate by more than 75 parsecs from the galactic plane, although there are rather larger deviations in the outermost parts of the Galaxy. In contrast to this arrangement about the galactic plane the distribution in the plane shows great irregularities. The hydrogen outlines the spiral arms of the Galaxy. The Sun is situated near the inner edge of one arm; the latter probably contains the Orion association, and the whole arm is now called the Orion arm. Outside this, at a distance from the galactic centre of between 10 and 12 kilo- 
parsecs, there is a conspicuous arm, called the Perseus arm, which contains the double cluster in Perseus. Inside the Orion arm, between 6 and 7 kiloparsecs from the centre, there is the Sagittarius arm. The hydrogen disk eppears to terminate at about 15 kiloparsecs from the centre. The maximum density of hydrogen in the spiral arms is about 2 atoms $/ \mathrm{cm}^{3}$; between the arms the density may fall to less than 0.05 atoms $/ \mathrm{cm} .^{3}$. These results are all compatible with the classification of the Galaxy as a spiral nebula of Hubble's type $S b$. It is estimated that about 2 per cent of the total mass of the Galaxy exists in the form of interstellar gas. In the central region there is an arm at a distance from the centre of 3 kiloparsecs. The arm passes between the Sun and the centre, and in addition to participating in galactic rotation, this arm appears to be expanding with a velocity of $53 \mathrm{~km}$. $/ \mathrm{sec}$. away from the centre. This arm can be seen in absorption against the source Sagittarius $A$ situated at the galactic centre. The existence of this expanding arm (and of other smaller features which are expanding) renders the rotation curve quite uncertain near the galactic centre.

The astounding flatness of the galactic interstellar medium is of importance in connexion with the internal motions in the medium. It provides a measure of the average random motion of the interstellar clouds, and it indicates that there must have been a considerable exchange of momentum from the inner to the outer parts of the disk. A fundamental question is whether spiral structure is a problem of gas dynamics (and magnetic fields) or of stellar dynamics. There seems no doubt that the spiral structure of galaxies is elosely related to their content of interstellar gas, and the latter may be a prerequisite for spiral structures to develop. Supergiants and other young stars are situated in the spiral arms; there is as yet little evidence relating to the distribution of older stars and gas. The Galaxy rotates in a period of about 200 million years, and the effects of differential galactic rotation would severely distort any existing arms. The arms must either be smoothed out and replaced within this time or be maintained by some unknown mechanism independently of galactic rotation. There are reasons for believing that interstellar magnetic fields must be involved in such a mechanism. Interstellar polarization of the light of distant stars indicates the presence of magnetic fields, in many cases along the spiral arms. Part of the continuous radio emission from the Galaxy is closely concentrated in the galactic plane, and must be connected with spiral structure; the radiation appears to be of non-thermal origin, and may originate in synchrotron radiation from electrons moving in interstellar magnetic fields. The existence of radio coronae of the Galaxy and of the Andromeda nebula also supports the idea of the importance of magnetic fields. Nevertheless, we are forced to the conclusion that we have made little progress towards understanding the origin of spiral structure. We have, however, a much-improved knowledge of what has to be explained.
R. H. Garstang

\title{
IODINE AND COPPER UPTAKE BY SOIL ORGANIC MATTER
}

\author{
By J. M. NORTHMORE
}

Scott Agricultural Laboratories, Department of Agriculture, Kenya

\begin{abstract}
$\mathrm{A}$ LTHOUGH the soil organo-metallic complex concept of Bremner et al. ${ }^{1}$ has been confirmed by the work of Beckwith ${ }^{2}$, these workers do not specify the nature of the organic component of the complex. However, the relationship found between the replacement capacity of soil organic matter and its phenolic hydroxyl group content ${ }^{3}$ points to material derived from lignin containing these groups being the organic component concerned. These considerations have led to an investigation of the relationships between the phenolic hydroxyl group content and the organo-metallic complex-forming capacity of the organic matter of a number of limed and unlimed soils from the Kenya Highlands. Wide ranges of $p \mathrm{H}$ values and organic matter contents were covered.

The phenolic hydroxyl group contents of the organic fractions of the soils were measured by the iodine uptakes according to the hypoiodite method of Norman and Peevy ${ }^{4}$ as modified by Moodie ${ }^{5}$. The eapacities of the same organic fractions to form organo-metallic complexes were measured by their copper uptakes by Lees's mothod ${ }^{6}$ slightly modified.

Copper was taken as the complexing metal as it is known to have a greater ability to compete for sorption sites on organic matter than most of the other bivalent metals present in soil ${ }^{2,7}$. Preliminary investigations of the copper uptakes have indicated
\end{abstract}

the possibility that added copper is preferentially absorbed by the organic fraction of a soil, and it is intended to offer evidence of this at a later date. Table 1 gives the copper and iodine uptake values obtained for the twelve soils studied.

The results show that increasing amounts of copper are taken up by the soil organic fractions with increasing $p \mathrm{H}$ values, the relationship between the two variables being curvilinear. No correlation

Table 1. Iodine and Copper Uptake by SOIL ORganic MatTer

\begin{tabular}{|c|c|c|c|c|c|}
\hline Soil & $\begin{array}{c}\text { Total } \\
\text { carbon } \\
\text { (per } \\
\text { cent) }\end{array}$ & Lime applied & $p \mathrm{H}$ & $\mid \begin{array}{c}\text { Copper } \\
\text { uptake } \\
\text { (m.equiv. } / \\
\text { gm. C) }\end{array}$ & $\begin{array}{c}\text { Iodine } \\
\text { uptake } \\
\text { (m.equiv. } \\
\text { gm. C) }\end{array}$ \\
\hline $\begin{array}{l}K 27 \\
K 20\end{array}$ & $\begin{array}{l}2 \cdot 76 \\
2 \cdot 86\end{array}$ & $\begin{array}{c}\text { No lime } \\
4,400 \text { lb /acre } \\
\text { in } 1953\end{array}$ & $\begin{array}{l}4 \cdot 74 \\
5 \cdot 16\end{array}$ & $\begin{array}{l}0.215 \\
0.268\end{array}$ & $\begin{array}{l}5 \cdot 19 \\
4 \cdot 55\end{array}$ \\
\hline$K 5$ & $2 \cdot 86$ & in 1953 & $5 \cdot 45$ & 0.304 & $4 \cdot 72$ \\
\hline$K 48$ & $2 \cdot 53$ & $\begin{array}{l}\operatorname{in} 1953 \\
17,740 \mathrm{lb} / \\
\text { acre in } 1953\end{array}$ & $6 \cdot 12$ & $0 \cdot 735$ & $4 \cdot 35$ \\
\hline $\begin{array}{l}M 6 \\
M 8\end{array}$ & $\begin{array}{l}4 \cdot 29 \\
4 \cdot 22\end{array}$ & $\begin{array}{c}\text { No lime } \\
15,000 \mathrm{lb} . / \\
\text { acre in } 1954\end{array}$ & $\begin{array}{l}4 \cdot 96 \\
6 \cdot 01\end{array}$ & $\begin{array}{l}0.221 \\
0.628\end{array}$ & $\begin{array}{l}4 \cdot 97 \\
4 \cdot 76\end{array}$ \\
\hline $\begin{array}{l}M F \\
M G 38 \\
M G 36 \\
M L 19 \\
M L 16 \\
E 127\end{array}$ & $\begin{array}{l}4 \cdot 31 \\
3 \cdot 58 \\
5 \cdot 62 \\
5 \cdot 39 \\
4 \cdot 93 \\
2 \cdot 44\end{array}$ & $\begin{array}{l}\text { No lime } \\
\text { No lime } \\
\text { No lime } \\
\text { No lime } \\
\text { No lime } \\
\text { No lime }\end{array}$ & $\begin{array}{l}5 \cdot 63 \\
5 \cdot 92 \\
5 \cdot 74 \\
5 \cdot 38 \\
5 \cdot 47 \\
5 \cdot 45\end{array}$ & $\begin{array}{l}0.418 \\
0.708 \\
0.386 \\
0.290 \\
0.382 \\
0.409\end{array}$ & $\begin{array}{l}5 \cdot 55 \\
5 \cdot 63 \\
4 \cdot 86 \\
5 \cdot 35 \\
5 \cdot 49 \\
5 \cdot 62\end{array}$ \\
\hline
\end{tabular}

